\title{
Tumor-suppressive function of long noncoding RNA MALAT1 in glioma cells by downregulation of MMP2 and inactivation of ERK/MAPK signaling
}

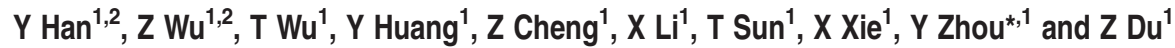

\begin{abstract}
Metastasis-associated lung adenocarcinoma transcript 1 (MALAT1) is a type of long noncoding RNA. It is associated with metastasis and is a favorable prognostic factor for lung cancer. Recent studies have shown that MALAT1 plays an important role in other malignancies. But, little is known about the role of MALAT1 in glioma. In this study, quantitative reverse transcription PCR (qRT-PCR) was used to demonstrate that the expression of MALAT1 was lower than that in normal brain tissues. Stable RNA interference-mediated knockdown of MALAT1 in human glioma cell lines (U87 and U251) significantly promoted the invasion and proliferation of the glioma cells by in vitro assays. Conversely, overexpression of MALAT1 caused significant reduction in cell proliferation and invasion in vitro, and tumorigenicity in both subcutaneous and intracranial human glioma xenograft models. Furthermore, MALAT1-mediated tumor suppression in glioma cells may be via reduction of extracellular signal-regulated kinase/ mitogen-activated protein kinase (ERK/MAPK) signaling activity and expression of matrix metalloproteinase 2 (MMP2). In conclusion, overall data demonstrated the tumor-suppressive role of MALAT1 in glioma by attenuating ERK/MAPK-mediated growth and MMP2-mediated invasiveness.
\end{abstract}

Cell Death and Disease (2016) 7, 2123; doi:10.1038/cddis.2015.407; published online 3 March 2016

Glioma is one of the most common types of primary brain tumors in adults, and represents one of the most aggressive and lethal human cancer types. ${ }^{1}$ Despite some advances in early detection, most of the patients are at advanced stages at the time of diagnosis, and the prognosis of these patients still remains poor. ${ }^{2}$ Although intensive research has been performed to detect and validate a number of molecules associated with glioma cell invasion and cell proliferation, only a few molecular mechanisms have been revealed and translated into clinical application so far.

Long noncoding RNAs (IncRNAs) are types of transcriptional products of the eukaryotic genome comprising $>200 \mathrm{nt}$ in length., In recent years, some IncRNAs have been found to be involved in carcinogenesis and cancer progression. Metastasis-associated lung adenocarcinoma transcript 1 (MALAT1) is one of the first found cancer-associated IncRNAs, and is also referred to as nuclear-enriched abundant transcript 2 (NEAT2). ${ }^{5}$ MALAT1 was initially found to be highly expressed in lung cancer and is a favorable prognostic factor for the survival of patients with stage I non-small-cell lung cancer (NSCLC). ${ }^{6}$ However, little is known about the role of MALAT1 in glioma progression. The prognosis of human glioma is poor, and the highly invasive nature of the disease represents a major impediment to current therapeutic modalities. ${ }^{7}$ At the molecular level, tumor cell invasion is mediated by a set of factors that initiate or promote cell motility, destruction of matrix, angiogenesis, and other biological events. $^{8-11}$ Extracellular signal-regulated kinase/mitogenactivated protein kinase (ERK/MAPK) signaling pathway mediates cell invasion and proliferation; aberrant activation of the ERK/MAPK signaling pathway has been observed in many types of human cancers including glioma. ${ }^{12-14}$ ERK/MAPK signaling orchestrates several key biological processes during the development and progression of cancer by inducing the transcription of a number of target genes regulating cell invasion. In particular, matrix metalloproteinase 9 (MMP9) and 2 (MMP2) levels increase with tumor progression in gliomas, and are thus known as key enzymes for invasion. ${ }^{15}$ In a previous study, it was found that MALAT1 promotes the proliferation and metastasis by activating the ERK/MAPK pathway. ${ }^{16}$ Hence this study investigated the expression of MALAT1 in glioma specimens and its regulation of glioma cell proliferation and invasion through the ERK/ MAPK signaling pathway and expression/activation of MMP.

\section{Results}

Expression of MALAT1 in glioma. To assess the expression of MALAT1 in noncancerous brain tissues and in

\footnotetext{
${ }^{1}$ Neurosurgery \& Brain and Nerve Research Laboratory, The First Affiliated Hospital of Soochow University, Suzhou, Jiangsu, PR China

${ }^{*}$ Corresponding author: Y Zhou, Department of Neurosurgery \& Brain and Nerve Research Laboratory, The First Affiliated Hospital of Soochow University, 188 Shizi Street, SuZhou 215006, Jiangsu, China. Tel: +86 0512 67781170; Fax: +86 051267781170; E-mail: zhouyouxin@suda.edu.cn

${ }^{2}$ These authors contributed equally to this work.

Abbreviations: MALAT1, metastasis-associated lung adenocarcinoma transcript 1; qRT-PCR, quantitative reverse transcription PCR; ERK/MAPK, extracellular signalregulated kinase/mitogen-activated protein kinase; MMP2, matrix metalloproteinase 2; MMP9, matrix metalloproteinase 9; IncRNAs, long noncoding RNAs; NEAT2, nuclear-enriched abundant transcript 2; NSCLC, non-small-cell lung cancer; DMEM, Dulbecco's modified Eagle's medium; FBS, fetal bovine serum; shRNA, short hairpin RNA; CMV, cytomegalovirus; GAPDH, glyceraldehyde-3-phosphate dehydrogenase; CCK-8, Cell Counting Kit-8; HRP, horseradish peroxidase; HOTAIR, HOX antisense intergenic RNA; CUDR, cancer upregulated drug resistant; HCC, hepatocellular carcinoma; GPC6, Glypican 6; CXCL5, C-X-C motif chemokine 5 Received 05.9.15; revised 05.12.15; accepted 09.12.15; Edited by E Candi
} 
different grades of glioma, the expression of MALAT1 was detected in 20 non-neoplastic brain tissues and in 132 human glioma tissue samples using quantitative reverse transcription PCR (qRT-PCR). Data showed that the expression of MALAT1 in noncancerous brain tissues was higher than in human brain glioma tissues $(P<0.01)$, but there was no obvious difference between different degrees of malignancy in glioma (low-grade versus high-grade gliomas) (Figure 1a). To determine the effect of MALAT1 in glioma, seven glioma cell lines (including three subculture lines carrying features of neural stem-like cell) were examined. As shown in Figure 1b, glioma stem cell lines of U87, SHG44 and SHG139 expressed higher levels of MALAT1 than their parental lines. U251 expressed lower levels of MALAT1 than that of U87. U251 and U87 were used in the present study to show the effect of overexpression of MALAT1, in addition to shRNA-mediated knock down.

Effects of MALAT1 shiRNA and overexpressing vector on the expression of MALAT1 in U87 and U251. MALAT1 was lowly expressed in glioma tissues, hence the function of MALAT1 was investigated using siRNA and overexpression vector in two glioma cell lines (U87 and U251). The lentiviral transfection efficiencies of the U87 and U251 cells were determined by examining the expression of GFP under a microscope $72 \mathrm{~h}$ after transfection. The efficiency of lentiviral transfection in both of the U87 and U251 cells was higher than $90 \%$ (Figure 2a). The expression of MALAT1 in U87 and U251 cells was examined using real-time PCR analysis after transfection with lentivirus. The levels of expression of MALAT1 in the U87 and U251 cells transfected with MALAT1 overexpression vector were increased by 3.65 -folds $(P<0.01)$ and 4.40 -folds $(P<0.01)$, respectively, compared with the control cells (Figures $2 b$ and $c$ ), while the levels of expression of MALAT1 in the U87 and U251 cells transfected with MALAT1-siRNA lentivirus (si-MALAT1) were decreased by $76.0 \% \quad(P<0.01)$ and $74.9 \% \quad(P<0.01)$, respectively, compared with the control cells (Figures $2 \mathrm{~b}$ and $\mathrm{c}$ ).

\section{MALAT1-suppressed U87 and U251 cell proliferation in vitro. To investigate whether the overexpression of MALAT1 could influence the proliferation of glioma cells in vitro, CCK-8 assay was performed. Figures $3 a$ and $b$ show that the proliferation abilities of the U87 and U251 cells decreased significantly after overexpression of MALAT1, but downregulation of MALAT1 increased the proliferation ability. Later the expression of $\mathrm{Ki}-67$ by these glioma cells was tested. With the upregulation of MALAT1, the expression of $\mathrm{Ki}-67$ decreased, while downregulation of MALAT1 increased the expression of Ki-67 (Figures $3 \mathrm{c}-\mathrm{e}$ ). To understand the effects of MALAT1 on cell-cycle distribution, glioma cells were analyzed using flow cytometry. The results indicated that overexpression of MALAT1 decreased the percentage of S cells and increased the percentage of G0/G1 cells $(P<0.01$, respectively), while downregulation of MALAT1 in cells showed the opposite results (Figures $3 f-h$ ).}

MALAT1-suppressed U87 and U251 cell proliferation in vivo. To determine the effects of MALAT1 on glioma cell growth in vivo, MALAT1 overexpressed or control U87 cells
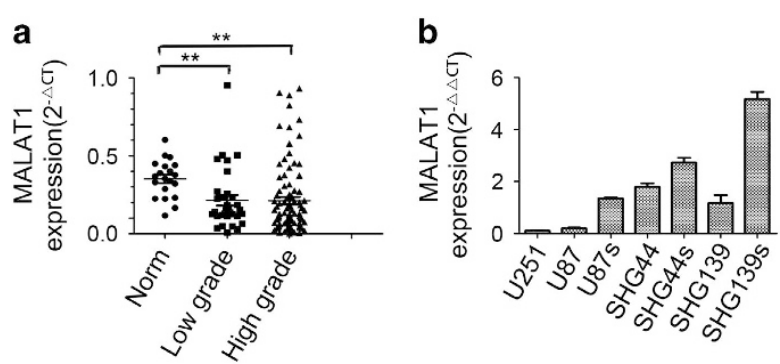

Figure 1 Expression of MALAT1 in glioma. (a) Expression of MALAT1 in human glioma tissues was lower than in noncancerous tissues $(P<0.01)$. (b) Expression of MALAT1 in human glioma cell lines. Abbreviation: MALAT1, metastasis-associated lung adenocarcinoma transcript 1

were injected into the left axilla of nude mice. As shown in Figures $4 a$ and $b$, the growth of tumors from the MALAT1-overexpressed xenografts of glioma cell line was significantly slower compared with that of tumors formed from the control cells $(P<0.01)$.

To assess the expression of MALAT1 in subcutaneous tumors and intracranial tumors, the expression of MALAT1 was detected in tumors formed by U87-negative cells and U87 cells transfected with MALAT1 vectors. Data showed that the expression of MALAT1 in tumors formed by U87 cells transfected with MALAT1 vectors was higher than U87-negative cells (Figures $4 \mathrm{c}$ and d) $(P<0.01)$.

The intracranial tumors were removed together with surrounding brain tissues prior to killing moribund mice normally shown 20-30\% weight loss, fixed, embedded and sectioned for immunohistochemical analysis. As shown in Figures $4 \mathrm{e}, \mathrm{h}$ and $\mathrm{i}$, intracranial xenografts of U87 cells transduced to overexpress MALAT1 had reduced proliferation index by Ki-67 staining and invasive potential by MMP2 staining compared with empty vector control. To investigate whether the ERK/MAPK pathway was affected in vivo, total protein was extracted from the intracranial tumors, and the results of western blotting showed that levels of phosphorylated ERK1/2 decreased in tumors formed by U87 cells transfected with MALAT1 vectors (Figures $4 \mathrm{f}$ and $\mathrm{g}$ ).

To further determine the effect of MALAT1 on glioma tumorigenicity in orthotopic location, MALAT1- or empty vector-infected U87 was implanted into nude mice frontal lobe of the brain. Overall survival data of mice demonstrated a significant effect of MALAT1 in suppressing the growth of U87-xenograft (Figure 4j).

Effects of upregulation and downregulation of MALAT1 on U87 and U251 cell invasiveness. To assess the effects of MALAT1 on the invasiveness of glioma cells, a transwell invasion system was used. The numbers of invasive cells with overexpressed MALAT1 were significantly reduced and the numbers of invasive cells with downregulated MALAT1 were increased compared with those of control cells (Figures $5 \mathrm{a}$ and $\mathrm{b}$ ). To further explore the mechanism of MALAT1 in suppressing glioma cell invasion, MMP2 and TIMP3 were examined using western blotting. Data showed that the expression level of MMP2 was markedly reduced due to the overexpression of MALAT1 and that the opposite result 

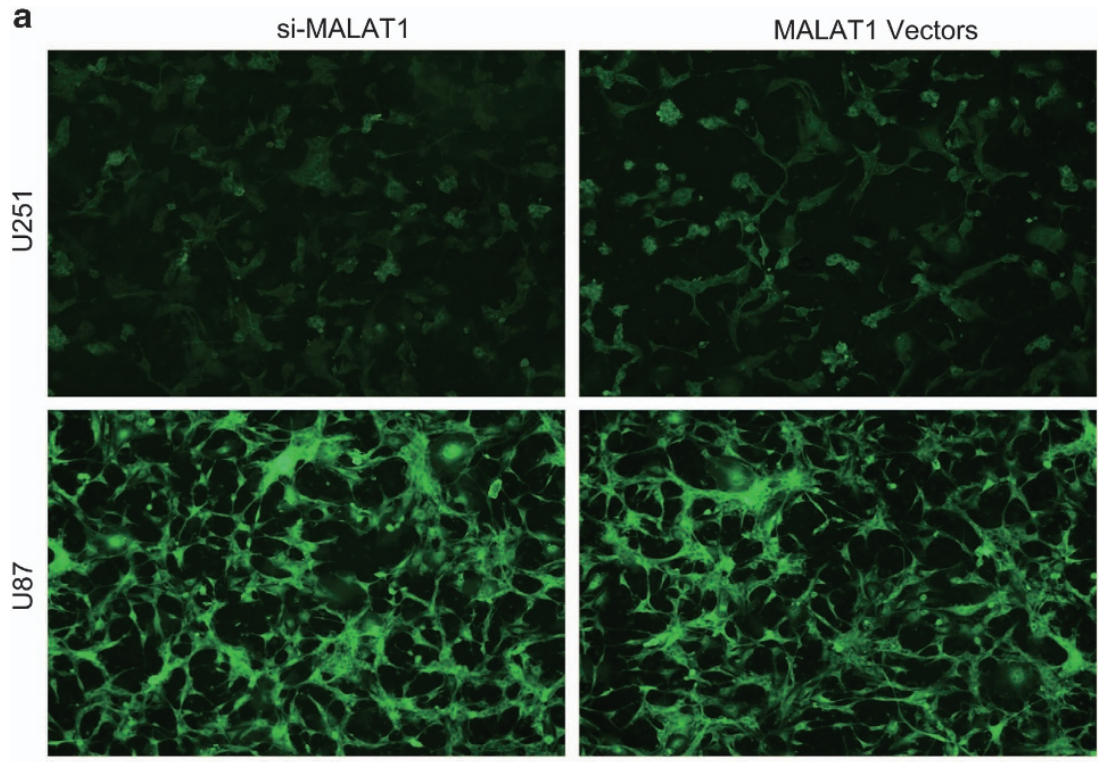

b

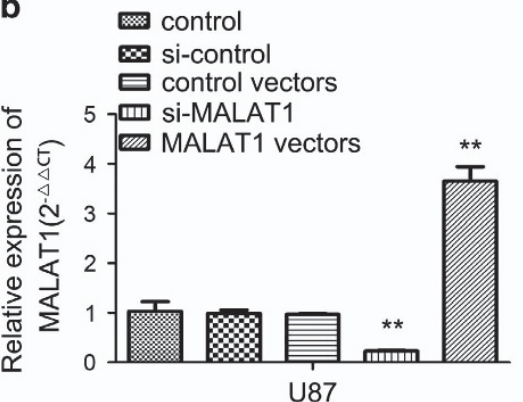

c

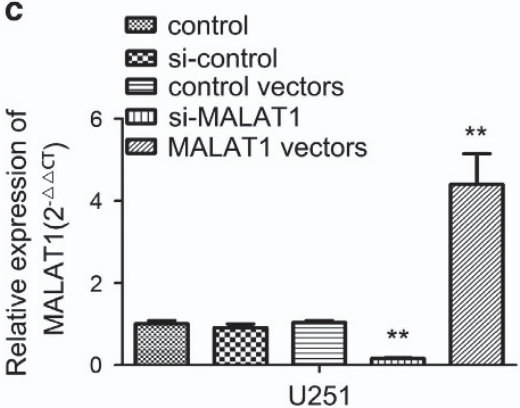

Figure 2 Effects of si-MALAT1- and MALAT1-overexpressing vectors on the expression of IncRNA MALAT1. (a) The transfection efficiency was determined 3 days after incubation with lentivirus at an $\mathrm{MOI}$ of 20 . The transfected cells labeled with GFP were observed under a fluorescence microscope ( $\times 200)$. (b and $\mathbf{c})$ Total RNA was extracted 4 days after infection, and the relative expression of MALAT1 was determined using quantitative real-time PCR. GAPDH was used as an internal control. The data represent the mean \pm S.D. of three independent experiments ( ${ }^{\star \star} P<0.01$ ). Abbreviations: GAPDH, glyceraldehyde-3-phosphate dehydrogenase; GFP, green fluorescence protein; IncRNA, long noncoding RNA; MALAT1, metastasis-associated lung adenocarcinoma transcript 1; MOI, multiplicity of infection; PCR, polymerase chain reaction; S.D., standard deviation

was observed by the knockdown of MALAT1 (Figures 5d and $5 e$ ). Gelatin zymography assays showed a single clear band at $\sim 67 \mathrm{kDa}$, indicating MMP2-mediated gelatin degradation, and this study confirmed similar changes at the protein level (Figure 5c). However, MALAT1 had little effect on the expression of TIMP3 (data not shown), further indicating that the mechanism of MALAT1-suppressed glioma cell invasion is via downregulation of expression of MMP2 at the protein level.

\section{Suppression of MAPK kinase pathways by overexpres-} sion of MALAT1. To determine the possible mechanism by which MALAT1 regulated the proliferation of glioma cells, western blot analysis was performed to investigate the effects of knockdown and overexpression of MALAT1 on the ERK/ MAPK pathway, which is often aberrantly activated in human cancers and contributes to enhanced cell proliferation and invasiveness. Western blot analysis showed that downregulation of MALAT1 significantly increased the levels of phosphorylated ERK1/2, and upregulation of MALAT1 reduced the levels of phosphorylated ERK $1 / 2$, while no detectable changes were observed in the total levels of ERK $1 / 2$ (Figures $6 a$ and c). To further verify the role of signaling activation and inactivation in MALAT1-aberrant expressed glioma cells, the addition of U0126 (a specific inhibitor of MEK/ERK) was used to pretreat cells. Results showed that with the presence of U0126, the upregulation of phosphorylated ERK1/2 and MMP2 by the knockdown of MALAT1 was attenuated. Consistently, the downregulation of phosphorylated ERK1/2 and MMP2 by the overexpression of MALAT1 was enhanced (Figures $6 b, d$ and e). These results indicate that MALAT1 regulates the ERK/MAPK signaling activity, which regulates MMP2 and overall glioma cell proliferation and invasion.

\section{Discussion}

Invasion and spread of solid gliomas are the major causes of death in patients with glioma. ${ }^{17}$ Therefore, identification of novel methods that can effectively inhibit the growth and invasion of gliomas is needed. The present study showed that IncRNA MALAT1 has a tumor-suppressive function in glioma 

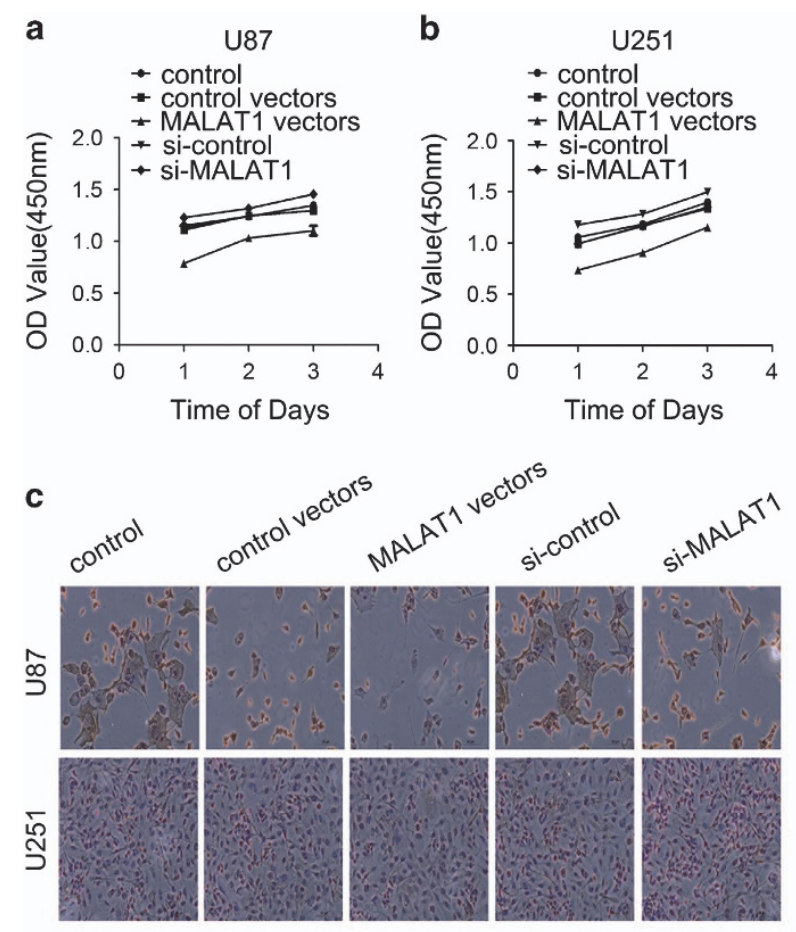

$\mathrm{f}$

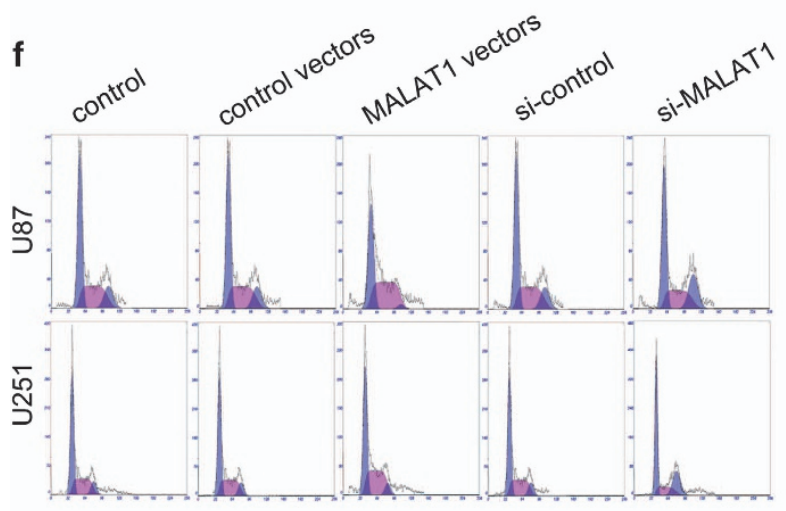

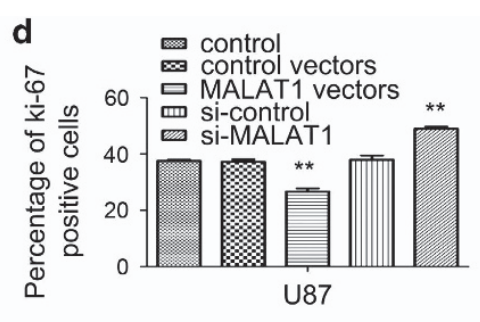
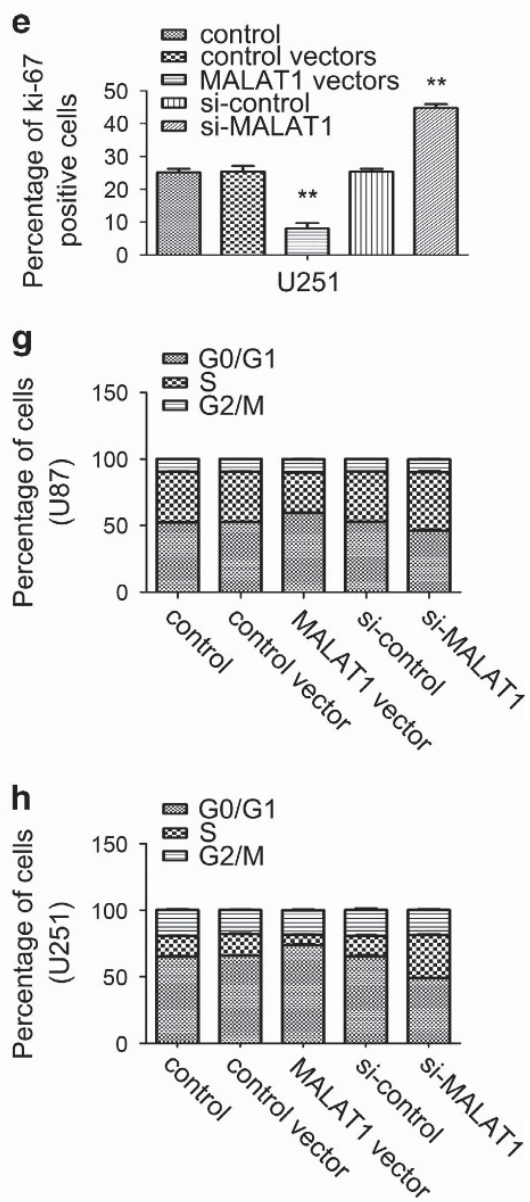

Figure 3 Effects of upregulation and downregulation of MALAT1 on U87 and U251 cell proliferation. (a and $\mathbf{b})$ Cellular proliferation of untransfected or transfected U87 and U251 cells was measured using a CCK-8 assay daily for 3 days. (c) Cellular proliferation of untransfected or transfected U87 and U251 cells was measured by testing the expression of Ki-67. (d and $\mathbf{e}$ ) The percentage of Ki-67-positive cells was calculated. Results are expressed as mean \pm S.D. from three independent experiments $(P<0.01)$. (f) Untransfected or transfected U87 and U251 cells were stained by propidium iodide and analyzed using flow cytometry. (g and $\mathbf{h})$ The percentage of cells in the G0/G1, S and G2/M phases of the cell cycle was calculated. Results are expressed as mean \pm S.D. from three independent experiments $(P<0.01)$. Abbreviations: CCK-8, Cell Counting Kit-8; MALAT1, metastasis-associated lung adenocarcinoma transcript 1; S.D., standard deviation

via suppressing both growth and invasion of cells, which is consistent with its lower expression levels in gliomas compared with normal brain tissues.

IncRNAs were initially considered to be spurious transcriptional noise, but recent evidence suggests that they may play a major biological role in cellular development and human diseases. ${ }^{18,19}$ IncRNAs such as HOTAIR (HOX antisense intergenic RNA), ANRIL (antisense noncoding RNA (ncRNA) in the INK4 locus), CUDR (cancer upregulated drug resistant) and MVIH (IncRNA associated with microvascular invasion in hepatocellular carcinoma (HCC)) have been shown to act as key molecules in the regulation of processes such as chromatin remodeling, transcription and post-transcriptional processing. ${ }^{20,21}$ MALAT1, which is also known as $\mathrm{HCN}$, NEAT2, PRO2853 and NCRNA00 047, is located at chromosome 11q13.1 and encodes a polyadenylated ncRNA of $\sim 8$ $\mathrm{kb}^{6,22} \mathrm{Ji}$ et al. found that MALAT1 was overexpressed in earlystage metastasizing NSCLC. High expression of MALAT1 was considered to be correlated with poor prognosis in patients with NSCLC. ${ }^{6}$ Therefore, MALAT1 has been proposed as a prognostic marker for metastasis and survival of patients with NSCLC. Additionally, Xie et al. $^{23}$ showed that MALAT1 was 


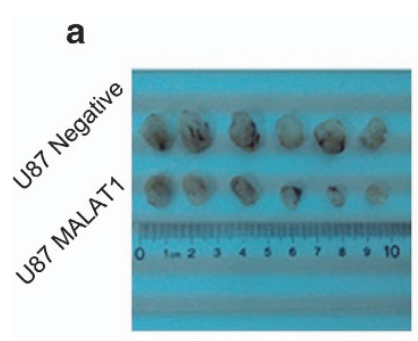

b
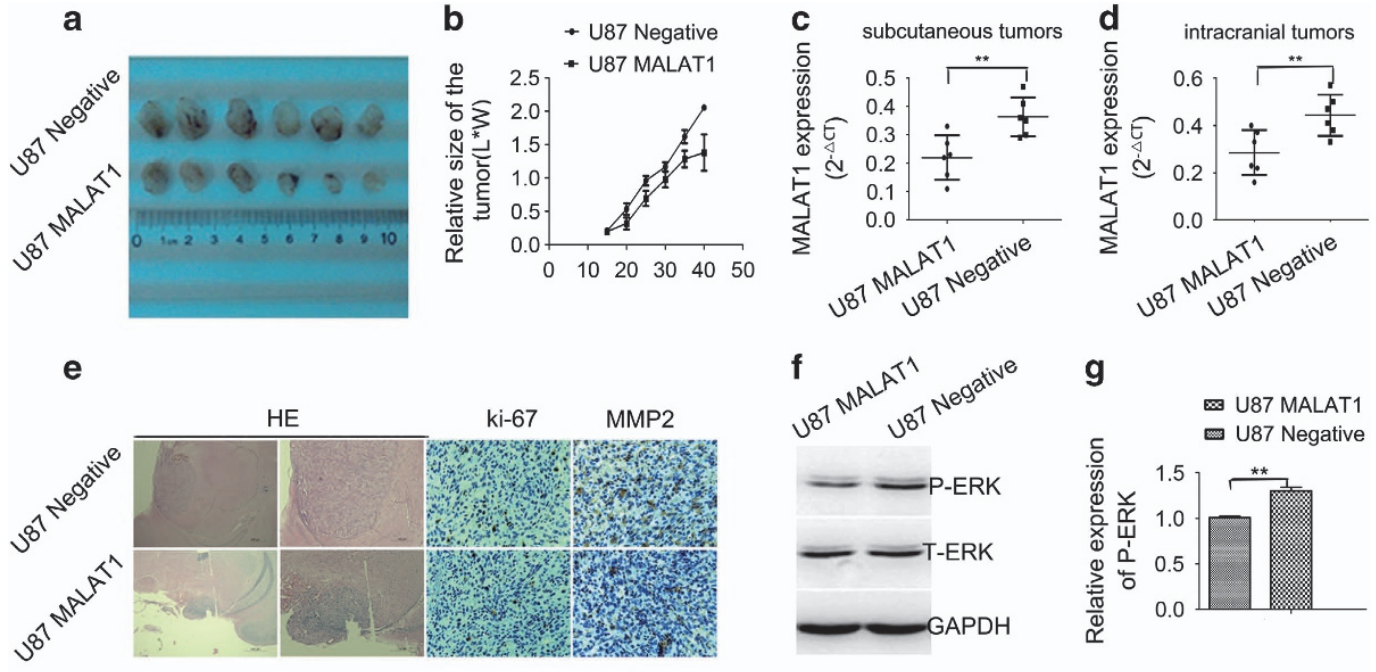

ki-67

MMP2

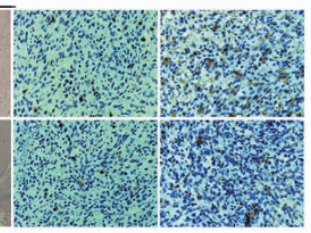

f

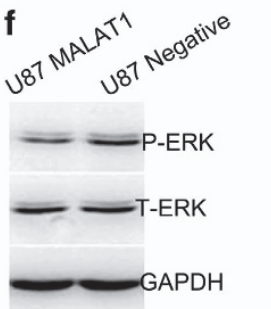

g

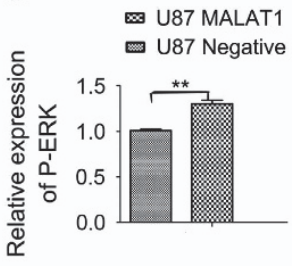

h

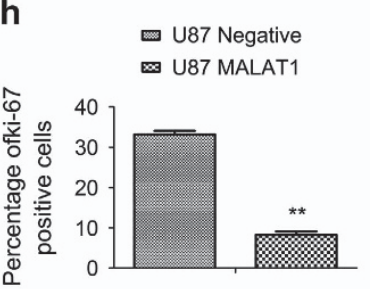

i

$\square$ U87 Negative

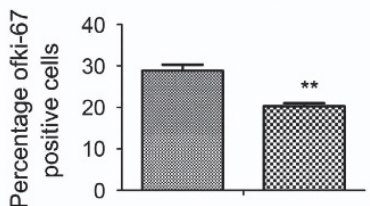

j

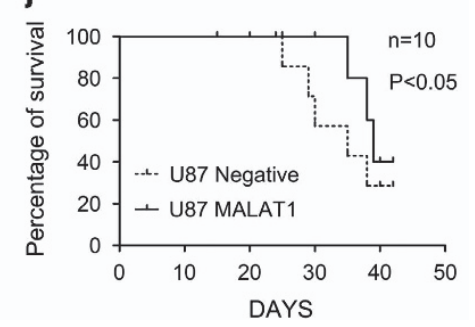

Figure 4 Effects of overexpression of MALAT1 on proliferation in vivo. (a and $\mathbf{b})$ Overexpression of MALAT1 reduced the growth of glioma in a subcutaneous glioma nude mouse model. (c and d) MALAT1 expression in subcutaneous and incratranial tumors $(P<0.01)$. (e) Immunohistochemistry showed that overexpression of MALAT1 reduced the expression of Ki-67 and MMP2 in intracranial xenograft of U87. ( $\mathbf{f}$ and $\mathbf{g})$ Western blotting showed that overexpression of MALAT1 reduced the expression of P-ERK $(P<0.01)$. ( $\mathrm{h}$ and i) The percentage of Ki-67- and MMP2-positive cells, respectively, calculated from densitometry immunohistochemistry signaling. Results are expressed as mean \pm S.D. from three independent experiments $(P<0.01)$. (j) Kaplan-Meier survival curves for nude mice implanted intracranially with U87 and its MALAT1 transfectants. MALAT1, metastasis-associated lung adenocarcinoma transcript 1

highly expressed in human nasopharyngeal carcinoma cell lines and can enhance the proliferation, invasion and metastasis of CNE-1 cells. Lai et al. ${ }^{24}$ found that MALAT1 is an independent prognostic factor for the recurrence of $\mathrm{HCC}$ after liver transplantation.

MALAT1 is commonly located in nuclear speckles. ${ }^{25}$ Nuclear speckle is an important subnuclear structure containing a large amount of nuclear proteins involved in precursor messenger mRNA alternative splicing and RNA transportation. ${ }^{26}$ This evidence supports the hypothesis that MALAT1 could be a regulator of post-transcriptional RNA processing or modification. Moreover, MALAT1 was shown to promote the invasion of cancer cell by inducing the expression of MMP9, and the activation of the ERK/MAPK pathway participates in this process. ${ }^{16}$ In addition, MALAT1 can also interact with the unmethylated form of CBX4, which controls the relocation of growth-control genes between the polycomb bodies and interchromatin granules, sites of silent or active gene expression, respectively. ${ }^{27}$

To elucidate the possible mechanism by which MALAT1 regulates the proliferation and invasion of glioma cell, western blot analysis of the key molecular factors of cancer-related pathways, such as nuclear factor kappaB, mTOR, Akt and others (data not shown), was performed. ${ }^{28-30}$ The ERK/MAPK pathway is one of the most important signal transduction pathways, and upregulation of MALAT1 inhibits the growth and invasion of tumor by inactivating this signaling cascade. It was observed that in U87 and U251 cells, overexpression of MALAT1significantly reduced the expression of phosphorylated ERK1/2. However, no detectable changes in the expression of total ERK1/2 protein were observed. To further verify the role of signaling activation and inactivation in MALAT1-aberrant expressed glioma cells, the addition of U0126 (a specific inhibitor of MEK/ERK) was used to pretreat cells. Results showed that inhibition of ERK1/2 signaling suppressed MALAT1 low expression-induced levels of phosphorylated ERK1/2 and MMP2.

The results of the present study were a little different from others. MALAT1 was shown to act as a tumor promoter gene in gallbladder cancer, lung cancer, colorectal cancer, etc. ${ }^{31-34}$ But in the present study, it was proved that MALAT1 acts as a tumor suppressor gene. It may be caused by the different types of tumors. And the direct link between the ERK/MAPK pathway and MALAT1 remains unclear. In previous studies, it has been proved that knockout of MALAT1 in lung cancer cells could significantly reduce the expression of several metastasis-related genes including Glypican 6 (GPC6) and $\mathrm{C}-\mathrm{X}-\mathrm{C}$ motif chemokine 5 (CXCL5), ${ }^{35,36}$ while depletion of 

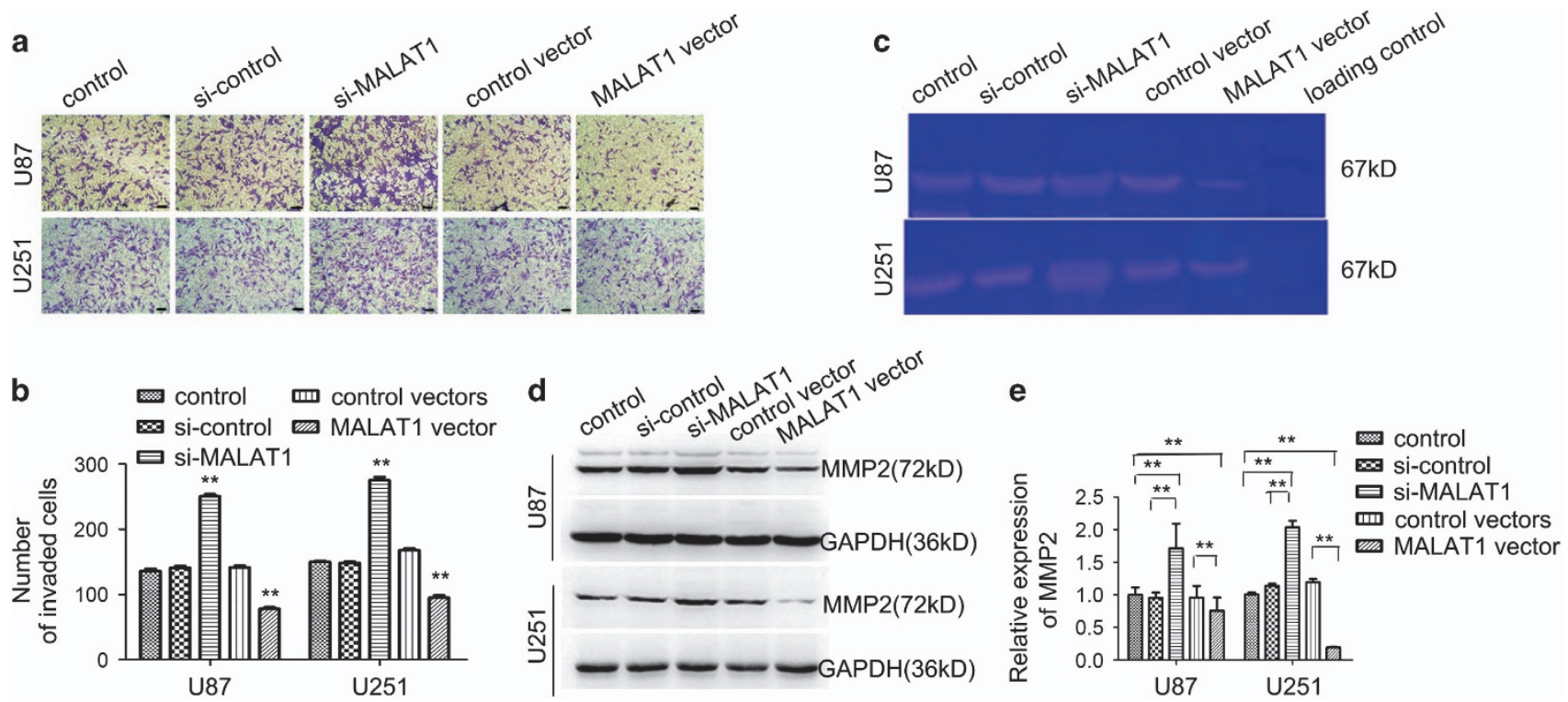

Figure 5 Effects of upregulation and downregulation of MALAT1 on glioma cell invasion in vitro. (a and $\mathbf{b}$ ) The transwell invasion system showed that upregulation of MALAT1 decreased the invasive cell numbers and downregulation of MALAT1 increased the invasive cell numbers compared with the cultures transfected with the negative control oligonucleotide. Each bar represents mean \pm S.D. from three independent experiments $(P<0.01)$. (c) Gelatin zymography assay showed that the overexpression of MALAT1 reduced the MMP2 protein and downregulation of MALAT1 increased the MMP2 protein secreted from cells. (d and e) The expression of MMP2 was obviously inhibited by the upregulation of MALAT1 and upregulated by the downregulation of MALAT1. Each bar represents mean \pm S.D. from six independent experiments $(P<0.01)$. MALAT1, metastasis-associated lung adenocarcinoma transcript 1; MMP2, matrix metalloproteinase 2; S.D., standard deviation

GPC6 or CXCL5 could lead to the inactivation of the MAPK pathway. Hence it was hypothesized that MALAT1 could probably inactivate the ERK/MAPK pathway via regulation of the expression of GPC6 or CXCL5 genes in glioma, which requires further study Overall data suggest tumor-suppressive effect of MALAT1 in glioma, by inhibiting the proliferation and invasion of glioma, and inactivates via regulation of the ERK MAPK pathway and expression of MMP2.

\section{Materials and Methods \\ Human tissue samples. For the study, 132 glioma samples were obtained from 132 Chinese patients (83 men and 49 women) between March 2011 and September 2013 from the Department of Neurosurgery, Brain and Nerve Research Laboratory of The First Affiliated Hospital of Soochow University. Two patients had grade I (pilocytic astrocytoma), 30 patients had grade II (diffuse astrocytoma), 60 patients had grade III (anaplastic astrocytoma) and 40 patients had grade IV (primary brain glioblastoma), according to the 2007 World Health Organization's classification system. The mean ages of the patients at the time of surgery were 49 years for men and 48.1 years for women. Twenty non-neoplastic brain tissue samples were obtained from adult patients with craniocerebral injuries, which required partial resections of brain tissue as decompression treatment to reduce intracranial pressure. All human samples were used in accordance with the policies of the institutional review board of The First Affiliated Hospital of Soochow University.}

Cell cultures. The human U87 and U251 cell lines were purchased from the Cell Bank Type Culture Collection of the Chinese Academy of Sciences (Shanghai, China). Cells were grown in Dulbecco's modified Eagle's medium (DMEM) (Hyclone; Thermo Fisher Scientific, Waltham, MA, USA) supplemented with 10\% fetal bovine serum (FBS) (GIBCO, Invitrogen Inc., Carlsbad, CA, USA).

Lentivirus-mediated RNA interference. The following short hairpin RNA (shRNA) 19 was used to target human MALAT1: sense: 5'-TGCTGTGTACTATCCCAT CACTGAAGGTTITGGCCACTGACTGACCTTCAGTGGGGATAGTACA-3'; antisense:

\section{5'-CCTGTGTACTATCCCCACTGAAGGTCAGTCAGTGGCCAAAACCTTCAGTGATG} GGATAGTACAC-3'.

The sequence of the negative control shRNA was Negative-F: tgctgAAATGT ACTGCGCGTGGAGACGTTTTGGCCACTGACTGACGTCTCCACGCAGTACATTT; Negative-R: cctgAAATGTACTGCGTGGAGACGTCAGTCAGTGGCCAAAACGTCTC CACGCGCAGTACATTTC.

These shRNAs were synthesized and inserted into the pFH1UGW lentivirus core vector containing a cytomegalovirus (CMV)-driven enhanced green fluorescent protein reporter gene; expression of the shRNA was driven by the $\mathrm{H} 1$ promoter. Recombinant lentivirus-expressing MALAT1-siRNA or control siRNA (si-MALAT1 or si-Control) was produced by Invitrogen Inc.

Lentiviral constructs. The sequence of MALAT1 was synthesized and subcloned into lentivector-transferred plasmid pCDH-CMV-MCS-EF1-coGFP to generate pCDH-CMV-MALAT1-EF1-COGFP. The recombinant vector PCDH-CMVMALAT1-EF1-COGFP or the control vector pCDH-CMVMCS-EF1-coGFP was triple transfected with the packaging vectors psPAX2 and pMD2.G into 293T cells using calcium chloride to produce the lentivirus by Invitrogen Inc. Glioma cells were infected with control or MALAT1-expressing lentivirus. The expression of MALAT1 in cells was determined using quantitative PCR.

Inhibition of expression of P-ERK1/2 by U0126 in cells. U87 and U251 cells were seeded onto a six-well plate and incubated at $37^{\circ} \mathrm{C}$ overnight. The cells were then treated with $10 \mathrm{mM}$ U0126 (Beyotime, Shanghai, China), a MAPK/ ERK kinase inhibitor that inhibits MEK $1 / 2$ for downregulation of expression of P-ERK for $72 \mathrm{~h}$. Then, the cells were infected with lentivirus.

Quantitative reverse transcription PCR. Total RNA from tissues and cells was isolated using TRIzol reagent (Invitrogen Inc.). Relative levels of MALAT1 IncRNA were examined using SYBRgreen real-time quantitative reverse transcription PCR (qRT-PCR) (Applied LightCycler480) and were normalized to levels of glyceraldehyde-3-phosphate dehydrogenase (GAPDH) mRNA. The following primers were used: InCRNA MALAT1 forward primer $5^{\prime}$-CTAAGGTCAAGAGAAGT GTCAG-3'; reverse primer 5'-AAGACCTCGACACCATCGTTAC-3'. GAPDH forward primer 5'-AACGGATTTGGTCGTATTG-3'; reverse primer 5'-GGAAGATGGTGAT 

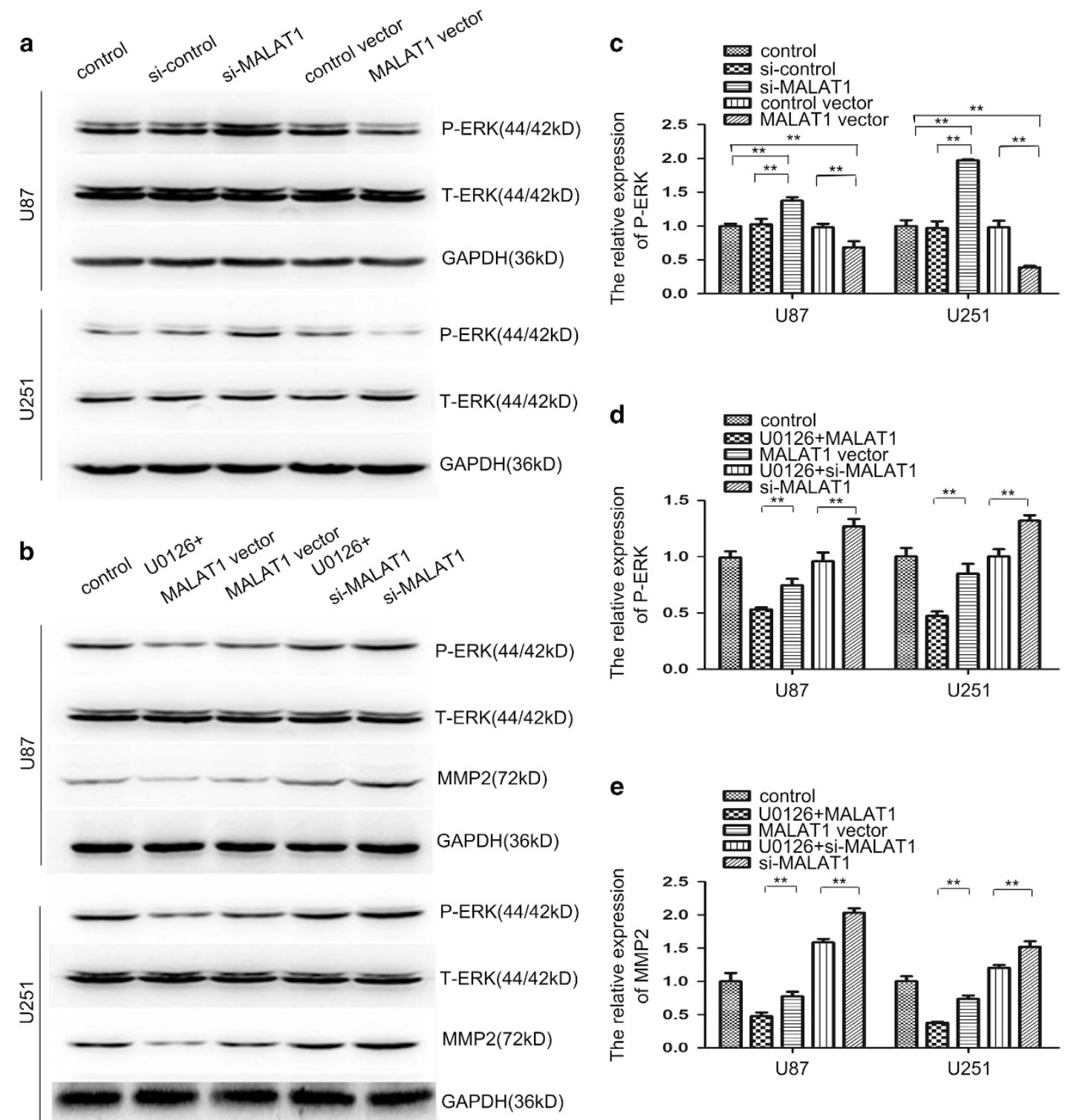

Figure 6 MALAT1 inactivated the ERK/MAPK pathway in the glioma cell lines. (a and $\mathbf{c}$ ) Western blot analysis showed that upregulation of MALAT1 reduced the expression of P-ERK and downregulation of MALAT1 increased its expression. Each bar represents the mean \pm S.D. from three independent experiments $\left({ }^{* \star} P<0.01\right)$. (b, $\mathbf{d}$ and $\left.\mathbf{e}\right)$ Western blot analysis showed that inhibition of ERK/MAPK signaling abrogated the downregulation of MALAT1-induced expression of P-ERK and MMP9 and promoted the effects of upregulation of MALAT1. Each bar represents the mean \pm S.D. from three independent experiments $\left({ }^{\star \star} P<0.01\right)$. ERK/MAPK, extracellular signal-regulated kinase/mitogenactivated protein kinase; MALAT1, metastasis-associated lung adenocarcinoma transcript 1; MMP, matrix metalloproteinase; S.D., standard deviation

GGGATT-3'. Relative expression was calculated using the $2^{-\triangle \triangle C T}$ method. All qRT-PCR analyses were performed in triplicate, and the data are presented as means \pm standard errors of the means.

Cell-cycle analysis. Both nontransfected and transfected U87 and U251 cells were harvested by trypsinization, washed three times in cold PBS and fixed in $70 \%$ ethanol at $4{ }^{\circ} \mathrm{C}$ overnight. After fixation, the cells were washed and resuspended in cold PBS and incubated in a solution of $10 \mathrm{mg} / \mathrm{ml}$ RNase and $1 \mathrm{mg} / \mathrm{ml}$ propidium iodide (Sigma-Aldrich, St. Louis, MO, USA) at $37^{\circ} \mathrm{C}$ for $30 \mathrm{~min}$ in the dark. Finally, the DNA content was determined using flow cytometry (BD Biosciences, Franklin Lake, NJ, USA). The percentage of cells in the G0/G1, $S$ and $\mathrm{G} 2 / \mathrm{M}$ phases was determined using Cell Quest acquisition software (BD Biosciences).
Cell proliferation assay. Cell proliferation was quantified using the Cell Counting Kit-8 (CCK-8; Beyotime). Briefly, $100 \mu$ l of cells from the five groups (control, vector control, vector-MALAT1, si-control and si-MALAT1) were seeded onto a 96-well plate at a concentration of 2000 cells per well and were incubated at $37^{\circ} \mathrm{C}$. At daily intervals (days 1, 2 and 3), the optical density was measured at $450 \mathrm{~nm}$ using a microtiter plate reader, and the rate of cell survival was expressed as the absorbance. The results represent the mean of six replicates under the same conditions.

Tumor cell matrigel invasion assay. Overall, $5 \times 10^{5}$ cells (U87 and U251) from each group were transferred on the top of Matrigel-coated invasion chambers (24-well insert, $8 \mu \mathrm{m}$ pore size; BD Biosciences) in serum-free DMEM. DMEM containing $0.05 \%$ FBS was added to the lower chamber as a chemoattractant. After an incubation period of $48 \mathrm{~h}$, noninvading cells were removed from the inner part of the insert using a cotton swab. Cells on the lower membrane 
surface were fixed in $4 \%$ formaldehyde and stained with $0.1 \%$ crystal violet. Invading cells were manually counted in five randomly chosen fields under a microscope, and photographs were taken.

Gelatin zymography. Equal numbers of glioblastoma cells/transfectants were plated in DMEM/F12 containing 5\% calf serum overnight to reach $80 \%$ confluence. Later culture medium was replaced with serum-free medium following rinsing of cells with PBS, and culture was continued for $48 \mathrm{~h}$. The conditioned medium was collected after removal of floating cells. Proteins in the medium were precipitated with four volumes of cold acetone, spun immediately at 14000 r.p.m. for 5 min at $4{ }^{\circ} \mathrm{C}$, and resuspended in radioimmunoprecipitation assay buffer containing $1 \times$ Protease Inhibitor Cocktail. Gelatin zymography was performed using equal amounts of protein $(0.5-1 \mu \mathrm{g}$ for U87 and $2-4 \mu \mathrm{g}$ for U251). Protein readings were based on the control for correct protein loading, and each experimental sample was repeated two to three times to verify the results.

Western blot analysis. The primary antibodies used were anti-MMP2 (Abcam, Tokyo, Japan), anti-TIMP3 (Abcam), anti-P-ERK1/2 and anti-T-ERK1/2 (Abcam). Protein samples were separated with $12 \%$ sodium dodecyl sulfatepolyacrylamide gel electrophoresis and transferred onto nitrocellulose membranes. Membranes were incubated with primary antibodies overnight at $4^{\circ} \mathrm{C}$. Membranes were washed and incubated for $2 \mathrm{~h}$ with horseradish peroxidase (HRP)-conjugated anti-rabbit secondary antibodies (Prosci Inc., Poway, CA, USA), followed by detection and visualization using electrochemiluminescence western blotting detection reagents (Pierce antibodies; Thermo Fisher Scientific).

Immunohistochemistry. Formalin-fixed paraffin-embedded U87 tumors were cut with a microtome into $6-\mu \mathrm{m}$ sections. Antigen retrieval was performed in $10 \mathrm{mM}$ sodium citrate buffer of $\mathrm{pH} 6$ for $16 \mathrm{~min}$ at $96-98^{\circ} \mathrm{C}$. Slides were incubated with primary antibodies against Ki-67 (Boster Bioengineering Co., Wuhan, China), and with antibodies against MMP2 and TIMP3 (Abcam). Sections were subsequently incubated with the Cell \& Tissue Staining Kit HRP-DAB system (R\&D Systems, Minneapolis, MN, USA), according to the manufacturer's instructions. Immunostaining was performed with known positive and negative tumor controls and were blindly evaluated by a pathologist.

Subcutaneous and intracranial implanted models. To explore the effects of MALAT1 on tumor growth and invasion in vivo, an intracranial and a subcutaneous nude mouse model was established using $1 \times 10^{5}$ and $1 \times 10^{6} \mathrm{U} 87$, respectively, cells infected with lentivirus-expressing MALAT1 or empty vector. On day 17 postimplantation, caliper measurements were performed to assess the growth of tumor. And animals with cells injected intracranially into the right frontal lobes of 4-6-week-old nu/nu mice brains were observed daily for neurologic symptoms. Those that were moribund were killed and that date was used to calculate survival.

Statistical analysis. Statistical analyses were performed using the SPSS software, Version 13.0 (SPSS, Chicago, IL, USA). Statistical significance was determined using two-tailed Student's $t$-test. A $P$-value of less than 0.05 was considered statistically significant.

\section{Conflict of Interest}

The authors declare no conflict of interest.

Acknowledgements. This work was supported by the National Natural Science Foundation of China (Grant No. 81372689) and Anti-Cancer Association Foundation of China (Grant No.CSNO-2013-MSD-009).

1. Taylor LP. Diagnosis, treatment, and prognosis of glioma: five new things. Neurology 2010 ; 75(Suppl 1): S28-S32.

2. Zhou Y, Liu F, Xu Q, Wang X. Analysis of the expression profile of Dickkopf-1 gene in human glioma and the association with tumor malignancy. J Exp Clin Cancer Res 2010; 29: 138

3. Rinn JL, Chang HY. Genome regulation by long noncoding RNAs. Ann Rev Biochem 2012; 81: $145-166$.

4. Mattick JS. The genetic signatures of noncoding RNAs. PLoS Genet 2009; 5: e1000459.

5. Gutschner T, Hammerle M, Diederichs S. MALAT1-a paradigm for long noncoding RNA function in cancer. J Mol Med (Berl) 2013; 91: 791-801.
6. Ji P, Diederichs S, Wang W, Boing S, Metzger R, Schneider PM et al. MALAT-1, a novel noncoding RNA, and thymosin beta4 predict metastasis and survival in early-stage nonsmall cell lung cancer. Oncogene 2003; 22: 8031-8041.

7. Yang TQ, Lu XJ, Wu TF, Ding DD, Zhao ZH, Chen GL et al. MicroRNA-16 inhibits glioma cell growth and invasion through suppression of BCL2 and the nuclear factor-kappaB1/MMP9 signaling pathway. Cancer Sci 2014; 105: 265-271.

8. Tektonidis M, Hatzikirou H, Chauviere A, Simon M, Schaller K, Deutsch A. Identification of intrinsic in vitro cellular mechanisms for glioma invasion. J Theor Biol 2011; 287: 131-147.

9. Goldbrunner RH, Bernstein JJ, Tonn JC. Cell-extracellular matrix interaction in glioma invasion. Acta Neurochir 1999; 141: 295-305; discussion 304-295.

10. Bikfalvi A, Moenner M, Javerzat S, North S, Hagedorn M. Inhibition of angiogenesis and the angiogenesis/invasion shift. Biochem Soc Trans 2011; 39: 1560-1564.

11. Jiang $\mathrm{L}$, Lin $\mathrm{C}$, Song $\mathrm{L}$, Wu J, Chen B, Ying $Z$ et al. MicroRNA-30e* promotes human glioma cell invasiveness in an orthotopic xenotransplantation model by disrupting the NF-kappaB/ IkappaBalpha negative feedback loop. J Clin Invest 2012; 122: 33-47.

12. Li B, Sun B, Zhu J, Zhou N, Yang Z, Gu J. Expression of RKIP in chronic myelogenous leukemia K562 cell and inhibits cell proliferation by regulating the ERK/MAPK pathway. Tumour Biol 2014; 35: 10057-10066.

13. Xu WH, Zhang JB, Dang Z, Li X, Zhou T, Liu J et al. Long non-coding RNA URHC regulates cell proliferation and apoptosis via ZAK through the ERK/MAPK signaling pathway in hepatocellular carcinoma. Int J Biol Sci 2014; 10: 664-676.

14. Kohsaka S, Hinohara K, Wang L, Nishimura T, Urushido M, Yachi $K$ et al. Epiregulin enhances tumorigenicity by activating the ERK/MAPK pathway in glioblastoma. Neurooncology 2014; 16: 960-970.

15. Forsyth PA, Wong H, Laing TD, Rewcastle NB, Morris DG, Muzik $H$ et al. Gelatinase-A (MMP-2), gelatinase-B (MMP-9) and membrane type matrix metalloproteinase-1 (MT1MMP) are involved in different aspects of the pathophysiology of malignant gliomas. $\mathrm{Br} \mathrm{J}$ Cancer 1999; 79: 1828-1835.

16. Wu XS, Wang XA, Wu WG, Hu YP, Li ML, Ding $Q$ et al. MALAT1 promotes the proliferation and metastasis of gallbladder cancer cells by activating the ERK/MAPK pathway. Cancer Biol Ther 2014; 15: 806-814.

17. Gong W, Wang Z, Wan $Y$, Shi L, Zhou Y. Downregulation of ABCG2 protein inhibits migration and invasion in U251 glioma stem cells. Neuroreport 2014; 25: 625-632.

18. Mercer TR, Dinger ME, Mattick JS. Long non-coding RNAs: insights into functions. Nat Rev Genet 2009; 10: 155-159.

19. Wilusz JE, Sunwoo H, Spector DL. Long noncoding RNAs: functional surprises from the RNA world. Genes Dev 2009; 23: 1494-1504.

20. Gutschner T, Diederichs $S$. The hallmarks of cancer: a long non-coding RNA point of view. RNA Biol 2012; 9: 703-719.

21. Yuan SX, Yang F, Yang Y, Tao QF, Zhang J, Huang G et al. Long noncoding RNA associated with microvascular invasion in hepatocellular carcinoma promotes angiogenesis and serves as a predictor for hepatocellular carcinoma patients' poor recurrence-free survival after hepatectomy. Hepatology 2012; 56: 2231-2241.

22. Hutchinson JN, Ensminger AW, Clemson CM, Lynch CR, Lawrence JB, Chess A. A screen for nuclear transcripts identifies two linked noncoding RNAs associated with SC35 splicing domains. BMC Genomics 2007; 8: 39.

23. Xie L, Hu Z, Wang X, Li Z. [Expression of long noncoding RNA MALAT1 gene in human nasopharyngeal carcinoma cell lines and its biological significance]. Nan Fang Yi Ke Da Xue Xue Bao= J South Med Univ 2013; 33: 692-697.

24. Lai MC, Yang Z, Zhou L, Zhu QQ, Xie HY, Zhang F et al. Long non-coding RNA MALAT-1 overexpression predicts tumor recurrence of hepatocellular carcinoma after liver transplantation. Med Oncol 2012; 29: 1810-1816.

25. Clemson CM, Hutchinson JN, Sara SA, Ensminger AW, Fox AH, Chess A et al. An architectural role for a nuclear noncoding RNA: NEAT1 RNA is essential for the structure of paraspeckles. Mol Cell 2009; 33: 717-726.

26. Lamond Al, Spector DL. Nuclear speckles: a model for nuclear organelles. Nat Rev Mol Cell Biol 2003; 4: 605-612.

27. Yang L, Lin C, Liu W, Zhang J, Ohgi KA, Grinstein JD et al. ncRNA- and Pc2 methylationdependent gene relocation between nuclear structures mediates gene activation programs. Cell 2011; 147: 773-788.

28. Zeng W, Chang H, Ma M, Li Y. CCL20/CCR6 promotes the invasion and migration of thyroid cancer cells via NF-kappa B signaling-induced MMP-3 production. Exp Mol Pathol 2014; 97 : 184-190.

29. Niu $H$, Wu B, Peng $Y$, Jiang $H$, Zhang $Y$, Wang $J$ et al. Erratum to: RNA interferencemediated knockdown of RhoGDI2 induces the migration and invasion of human lung cancer A549 cells via activating the PI3K/Akt pathway. Tumour Biol 2014; 36: 459.

30. Muscella A, Vetrugno C, Calabriso N, Cossa LG, De Pascali SA, Fanizzi FP et al. [Pt(O,O'acac)(gamma-acac)(DMS)] alters SH-SY5Y cell migration and invasion by the inhibition of $\mathrm{Na}+/ \mathrm{H}+$ exchanger isoform 1 occurring through a PKC-epsilon/ERK/mTOR pathway. PloS One 2014; 9: e112186.

31. Ji Q, Liu X, FuX, Zhang L, Sui H, Zhou L et al. Resveratrol inhibits invasion and metastasis of colorectal cancer cells via MALAT1 mediated Wnt/beta-catenin signal pathway. PloS One 2013; 8: e78700.

32. Shen L, Chen L, Wang $Y$, Jiang X, Xia H, Zhuang Z. Long noncoding RNA MALAT1 promotes brain metastasis by inducing epithelial-mesenchymal transition in lung cancer. $J$ neuro-oncol 2014; 121: 101-108. 
33. Wang J, Su L, Chen X, Li P, Cai $Q$, Yu B et al. MALAT1 promotes cell proliferation in gastric cancer by recruiting SF2/ASF. Biomed Pharmacother 2014; 68: 557-564.

34. Zheng HT, Shi DB, Wang YW, Li XX, Xu Y, Tripathi P et al. High expression of IncRNA MALAT1 suggests a biomarker of poor prognosis in colorectal cancer. Int J Clin Exp Pathol 2014; 7: 3174-3181.

35. Yiu GK, Kaunisto A, Chin YR, Toker A. NFAT promotes carcinoma invasive migration through glypican-6. Biochem J 2011; 440: 157-166.

36. Merabova N, Kaminski R, Krynska B, Amini S, Khalili K, Darbinyan A. JCV agnoprotein-induced reduction in CXCL5/LIX secretion by oligodendrocytes is associated with activation of apoptotic signaling in neurons. J Cell Physiol 2012; 227: 3119-3127. (c) (i) Cell Death and Disease is an open-access journal published by Nature Publishing Group. This work is licensed under a Creative Commons Attribution 4.0 International License. The images or other third party material in this article are included in the article's Creative Commons license, unless indicated otherwise in the credit line; if the material is not included under the Creative Commons license, users will need to obtain permission from the license holder to reproduce the material. To view a copy of this license, visit http://creativecommons.org/licenses/by/4.0/ 